\title{
Genetic analysis of a case of Helicobacter pylori-uninfected intramucosal gastric cancer in a family with hereditary diffuse gastric cancer
}

\author{
Taro Funakoshi ${ }^{1} \cdot$ Shin'ichi Miyamoto $^{2} \cdot$ Nobuyuki Kakiuchi $^{2} \cdot$ Mitsuhiro Nikaido $^{2} \cdot$ Takeshi Setoyama $^{3}$. \\ Akira Yokoyama ${ }^{1} \cdot$ Takahiro Horimatsu $^{1}$ - Atsushi Yamada ${ }^{1} \cdot$ Masako Torishima $^{4} \cdot$ Shinji Kosugi $^{4} \cdot$ Hidetaka Yamada $^{5}$. \\ Haruhiko Sugimura $^{5} \cdot$ Hironori Haga ${ }^{6} \cdot$ Yoshiharu Sakai $^{7} \cdot$ Seishi Ogawa ${ }^{8} \cdot$ Hiroshi Seno $^{2} \cdot$ Manabu Muto $^{1}$. \\ Tsutomu Chiba ${ }^{9}$
}

Received: 17 September 2018 / Accepted: 5 December 2018 / Published online: 12 December 2018

(c) The International Gastric Cancer Association and The Japanese Gastric Cancer Association 2018

\begin{abstract}
Germline mutations in $\mathrm{CDH}$, encoding E-cadherin, are known to be the causative mechanism of hereditary diffuse gastric cancer (HDGC). We encountered two cases of gastric cancer in a Japanese family with HDGC. A 28 -year-old man (Case 1) died of advanced gastric cancer. His younger sister aged 27 (Case 2) was diagnosed with intramucosal signet ring cell carcinoma (SRCC). Both had identical germline $\mathrm{CDH} 1$ mutations, but Case 1 was positive for Helicobacter pylori infection, whereas Case 2 was negative. Case 2 underwent total gastrectomy. Whole-exome sequencing of an intramucosal SRCC in Case 2 revealed seven somatic mutations including one in $C D H 1$. The six non- $C D H 1$ mutations were classified as non-driver mutations. Decreased expression of E-cadherin in intramucosal SRCC was confirmed by immunohistochemistry. Our report demonstrated that $\mathrm{CDH} 1$ mutation was the only active driver mutation in Helicobacter pylori-uninfected intramucosal SRCC.
\end{abstract}

Keywords Hereditary diffuse gastric cancer $\cdot$ Helicobacter pylori-uninfected gastric cancer $\cdot$ Signet ring cell carcinoma $\mathrm{CDHl} \cdot$ E-cadherin

Shin'ichi Miyamoto

shmiyamo@kuhp.kyoto-u.ac.jp

1 Department of Therapeutic Oncology, Graduate School of Medicine, Kyoto University, Kyoto, Japan

2 Department of Gastroenterology and Hepatology, Graduate School of Medicine, Kyoto University, 54, Shogoin-kawahara-cho, Sakyo-ku, Kyoto 606-8507, Japan

3 Department of Gastroenterology and Hepatology, Osaka Red Cross Hospital, Osaka, Japan

4 Department of Medical Ethics and Medical Genetics, Kyoto University School of Public Health, Kyoto, Japan

5 Department of Tumor Pathology, Hamamatsu University School of Medicine, Hamamatsu, Japan

6 Department of Diagnostic Pathology, Graduate School of Medicine, Kyoto University, Kyoto, Japan

7 Department of Surgery, Graduate School of Medicine, Kyoto University, Kyoto, Japan

8 Department of Pathology and Tumor Biology, Graduate School of Medicine, Kyoto University, Kyoto, Japan

9 Kansai Electric Power Hospital, Osaka, Japan

\section{Introduction}

Hereditary diffuse gastric cancer (HDGC) is an inherited form of gastric cancer. Approximately $40 \%$ of HDGC patients have germline mutations in $\mathrm{CDH} 1$, which encodes E-cadherin [1]. E-cadherin is a calcium-dependent cell membrane protein involved in cell-cell adhesion that is a known tumor suppressor. The cumulative risk of diffuse gastric cancer in HDGC patients with germline $\mathrm{CDH} 1$ mutations is approximately $70 \%$ for men and $56 \%$ for women [2]. The distinctive microscopic feature of early gastric cancer in family members with HDGC is multiple foci of intramucosal signet ring cell carcinoma (SRCC) [3-6]. Although the role of $\mathrm{CDH} 1$ mutations in HDGC has been well studied [1, 7], little is known about the genetic alterations in intramucosal SRCC.

Here, we present two patients with early-onset HDGC belonging to a Japanese family with HDGC. Whole-exome sequencing (WES) of an intramucosal SRCC from one patient was performed to clarify the carcinogenic mechanism of HDGC. 


\section{Case presentation}

\section{Case 1}

A 27-year-old man presented with abdominal pain. Esophagogastroduodenoscopy (EGD) revealed advanced infiltrative gastric cancer at the greater curvature of upper gastric body on a background of atrophic gastric mucosa (Fig. 1a, b). Histopathological examination using a biopsy specimen revealed poorly differentiated adenocarcinoma (Fig. 1c). Immunohistochemical analysis for Helicobacter pylori ( $\mathrm{Hp}$ ) was positive. Abdominal computed tomography demonstrated peritoneal dissemination (Fig. 1d). The patient was given a diagnosis of unresectable advanced gastric cancer. He received palliative chemotherapy but died of the disease 9 months after the initiation of chemotherapy.

\section{Case 2}

A 27-year-old woman (the younger sister of Case 1) underwent surveillance EGD and was found to have an intramucosal gastric cancer in the middle gastric body on a background of nonatrophic gastric mucosa (Fig. 2a, b, c, d). Serum antibody for $H p$ and immunohistochemical analysis for $H p$ were negative. She had no history of $H p$ eradication therapy. These results strongly suggested that she was $H p$-uninfected. She underwent total gastrectomy (Fig. 3).
The presence of intramucosal SRCC with no component of poorly differentiated adenocarcinoma (Fig. 3, lesion 1), which was detected by surveillance EGD, was confirmed by histology (Fig. 4a, b). In addition, two other intramucosal SRCCs that could not be detected before surgery were also found in the prepylorus (Figs. 2d, 3). Decreased expression of E-cadherin (Fig. 4c) and an absence of nuclear accumulation of P53 (Fig. 4d) of the lesion 1 were confirmed by immunohistochemistry.

\section{Genetic analysis}

\section{Presence of a germline $\mathrm{CDH} 1$ mutation}

The two patients (Cases 1 and 2) had several family members with a history of gastric cancer (Fig. 5), which according to the International Gastric Cancer Linkage Consortium clinical criteria for HDGC, raised the possibility that they were a family at risk of HDGC [8], especially because their father also had a history of gastric cancer. Therefore, the patients and their father were offered genetic counseling and were tested for $\mathrm{CDH} 1$ mutations. DNA was extracted from their peripheral blood. Combination of polymerase chain reaction-direct sequencing analysis and multiplex ligationdependent probe amplification was performed for all the exons of $\mathrm{CDH} 1$ as described previously [7]. This genetic testing revealed an identical germline $\mathrm{CDH} 1$ mutation (nonsense mutation at exon 3 , c. $187 \mathrm{C}>\mathrm{T}$; p.R63*) in both the
Fig. 1 Macroscopic and microscopic findings in Case 1. a Esophagogastroduodenoscopy revealed infiltrative ulcerative type of gastric cancer at the greater curvature of the upper gastric body. b Atrophic change was observed at the lesser curvature of the gastric body. c Histopathological examination of the biopsy specimen revealed poorly differentiated adenocarcinoma (hematoxylin and eosin staining, $\times 100)$. d Abdominal computed tomography demonstrated peritoneal dissemination (arrows)
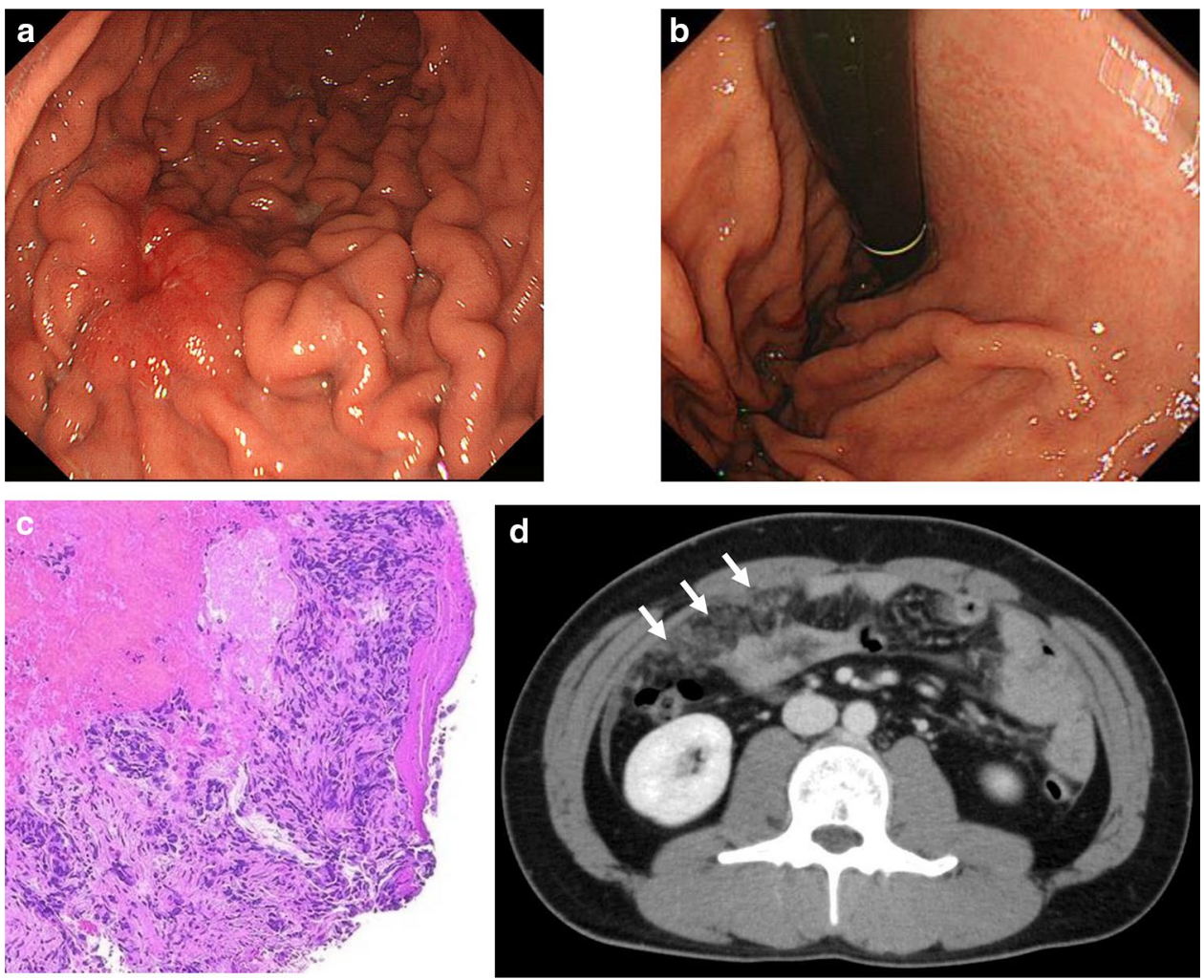
Fig. 2 Endoscopic findings in Case 2. a Esophagogastroduodenoscopy revealed a flat and discolored area at the greater curvature of the middle gastric body. $\mathbf{b}$ The demarcation line of the lesion was clear on narrowband imaging. $\mathbf{c}$ Esophagogastroduodenoscopy showed no atrophic change in the gastric body. d No abnormal findings were observed in the prepylorus (see Fig. 3)
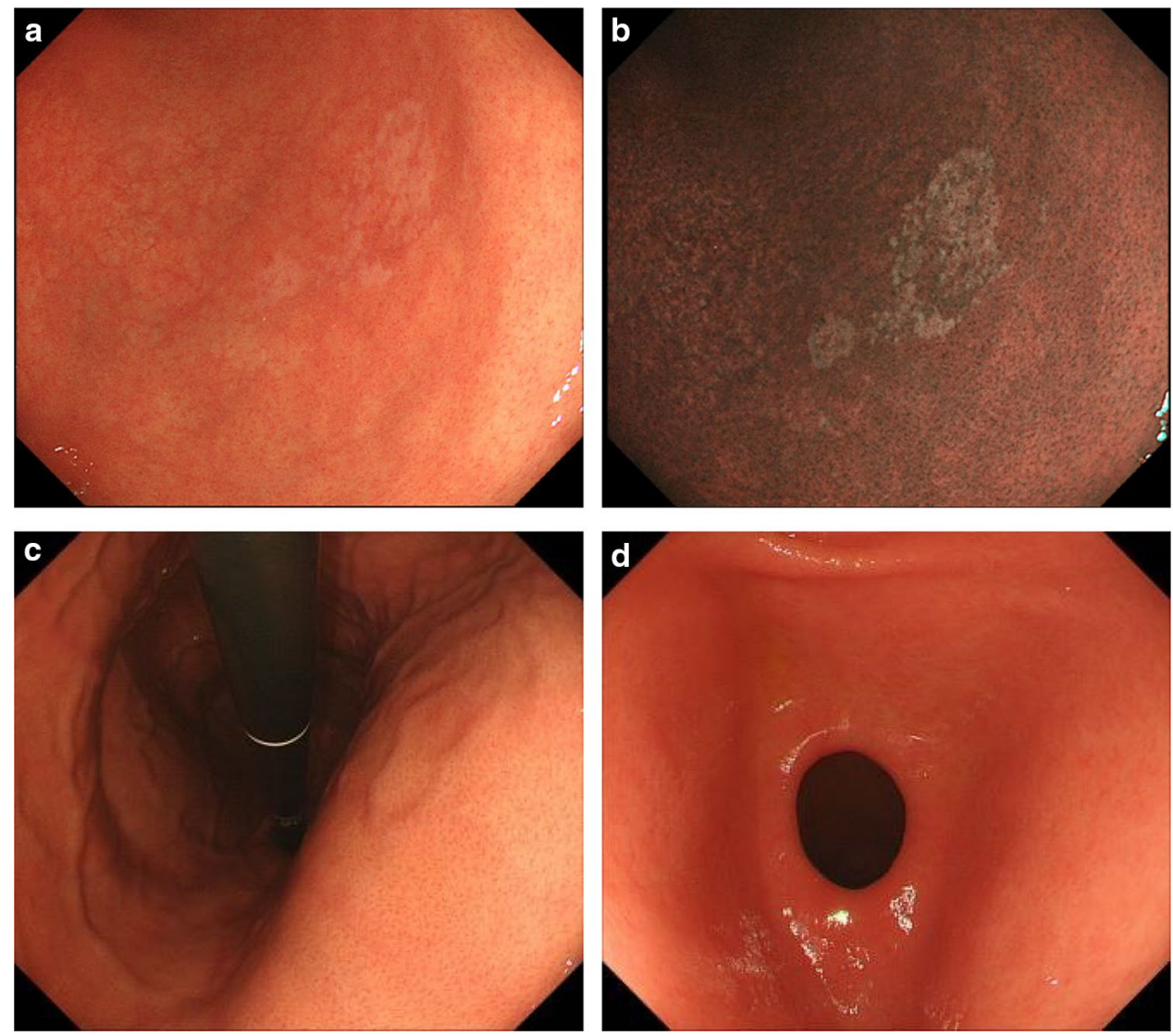

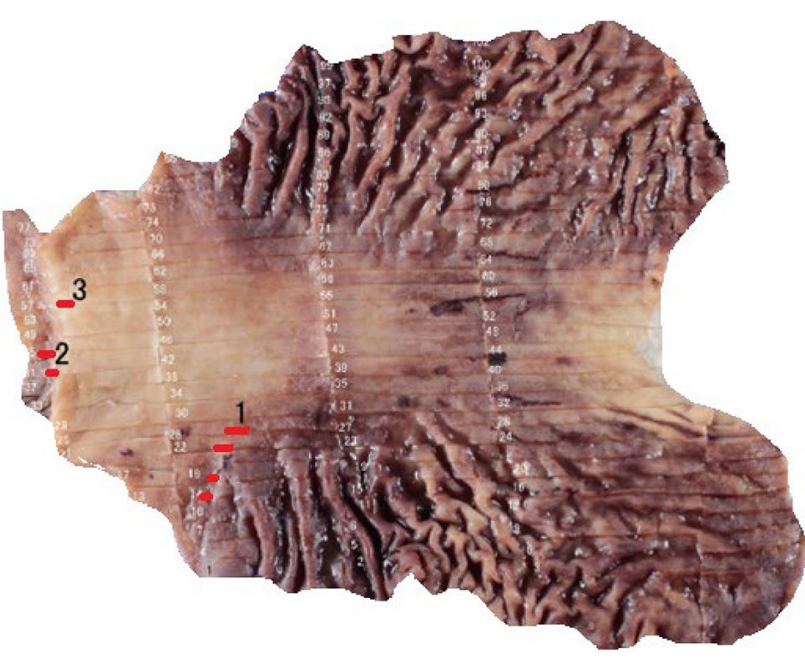

Fig. 3 Gastrectomy mapping study of Case 2. Red lines indicate foci of intramucosal SRCC. The main lesion is indicated by line 1 (lesion 1). Two other intramucosal SRCCs (lesion 2 and 3 indicated by line 2 and 3, respectively) that could not be detected before surgery were found in the prepylorus

patients and their father. This family had the strongest family history of gastric cancer compared to four HDGC families previously reported in Japan [5-7].

\section{Presence of a somatic $\mathrm{CDH} 1$ mutation in the intramucosal SRCC of Case 2}

To characterize the molecular basis of the development of SRCC, we performed WES of the resected specimen. Written informed consent was obtained according to protocols approved by the Ethics Committee of Kyoto University. Genomic DNA was isolated from the intramucosal SRCC (Fig. 3, lesion 1) in formalin-fixed paraffin-embedded tissue and from a matched peripheral blood sample using GeneRead DNA FFPE Kit and DNeasy Blood and Tissue Kit (Qiagen), respectively. DNA libraries were prepared using SureSelect Human All Exon V5 (Agilent Technologies) and were sequenced using an Illumina HiSeq 2500 platform. Mutation calling was performed using the Genomon pipeline (http:// genomon.hgc.jp/exome/en/index.html). Candidate mutations were validated by polymerase chain reaction-based amplicon deep sequencing using matched biopsy sample from the intramucosal SRCC [9]. The results identified seven somatic mutations; a $\mathrm{CDH} 1$ mutation (missense mutation at exon 5, c.641T >C; p.L214P) and other six non-driver mutations (Table 1), in addition to the germline mutation (nonsense mutation at exon 3, c. 187C>T; p.R63*) that was identical to those found in the DNA from peripheral blood of the two family members (Case 1 and their father). 

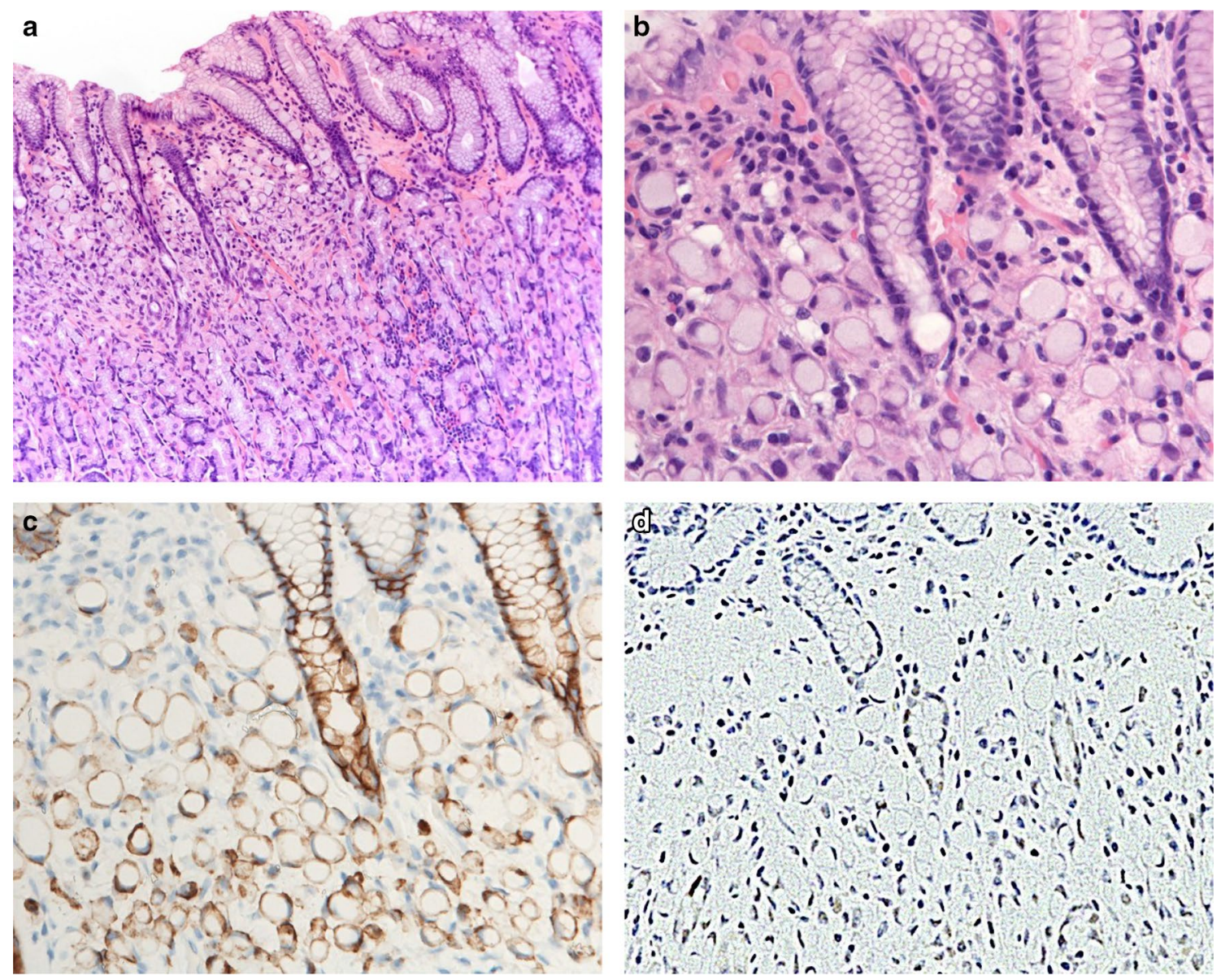

Fig. 4 Microscopic findings of a SRCC in Case 2 (lesion 1 in Fig. 3). Hematoxylin and eosin staining of the lesion $1(\mathbf{a} \times 100, \mathbf{b} \times 200$, respectively). SRCC was confined to the proliferative zone of the mucosa. c Immunohistochemistry for E-cadherin (Clone $\mathrm{NCH}-38$ )

$(\times 200)$. SRCC showed weak immunoreactivity. d Immunohistochemistry for P53 (Clone DO-7) showed no nuclear accumulation in SRCC $(\times 200)$

\section{Discussion}

To our knowledge, this is the first study showing the results of WES of the intramucosal SRCC specimen in family members with HDGC. Although the role of germline mutations of $\mathrm{CDH} 1$ in HDGC has been well studied, little is known about the genetic alterations in intramucosal SRCC, because these tumors are very small and it is difficult to obtain a sufficient amount of DNA.

We were able to perform WES of an intramucosal SRCC in Case 2, the results of which revealed a germline and a somatic mutation of $\mathrm{CDHl}$ that were both pathogenic: the germline mutation was a nonsense mutation of exon 3 [10], which encodes the precursor of E-cadherin, resulting in the expression of truncated non-functional protein. On the other hand, the somatic mutation was a missense mutation of exon 5, c.641T $>$ C and p.L214P, which has been reported in the analysis of gastric cancer [11, 12] and to affect protein function in vitro [13]. Although the possibility that these two

mutations are present on the same allele cannot be excluded, the somatic mutation could lead to inactivation of the protein encoded by the remaining wild-type $\mathrm{CDHI}$ allele through a second-hit mechanism, resulting in a loss of E-cadherin function. Indeed, immunohistochemical analysis confirmed that E-cadherin expression was impaired in the intramucosal SRCC contrasting to adjacent normal epithelium, suggesting loss of E-cadherin function.

However, our analysis showed other six mutations in addition to those in $\mathrm{CDH} 1$. These mutations have not been reported as active driver mutations in the comprehensive molecular analyses of gastric cancer [12, 14-17]. Moreover, they could not be identified in any other types of tumor during the search of the COSMIC v86 database (https://cancer.sange r.ac.uk/cosmic). These results strongly suggest that these six mutations are unlikely to be driver mutations.

We emphasize that only a small number of mutations were detected in the intramucosal SRCC. For example, there was no mutation in TP53, one of the most frequently 
Fig. 5 Family tree of this family with HDGC including Cases 1 and 2 . The shaded square and circle represent the family members with gastric cancer. $G C$ age at diagnosis of gastric cancer; $E+C D H 1$ mutation positive; $E-C D H 1$ mutation negative, $P$ Proband

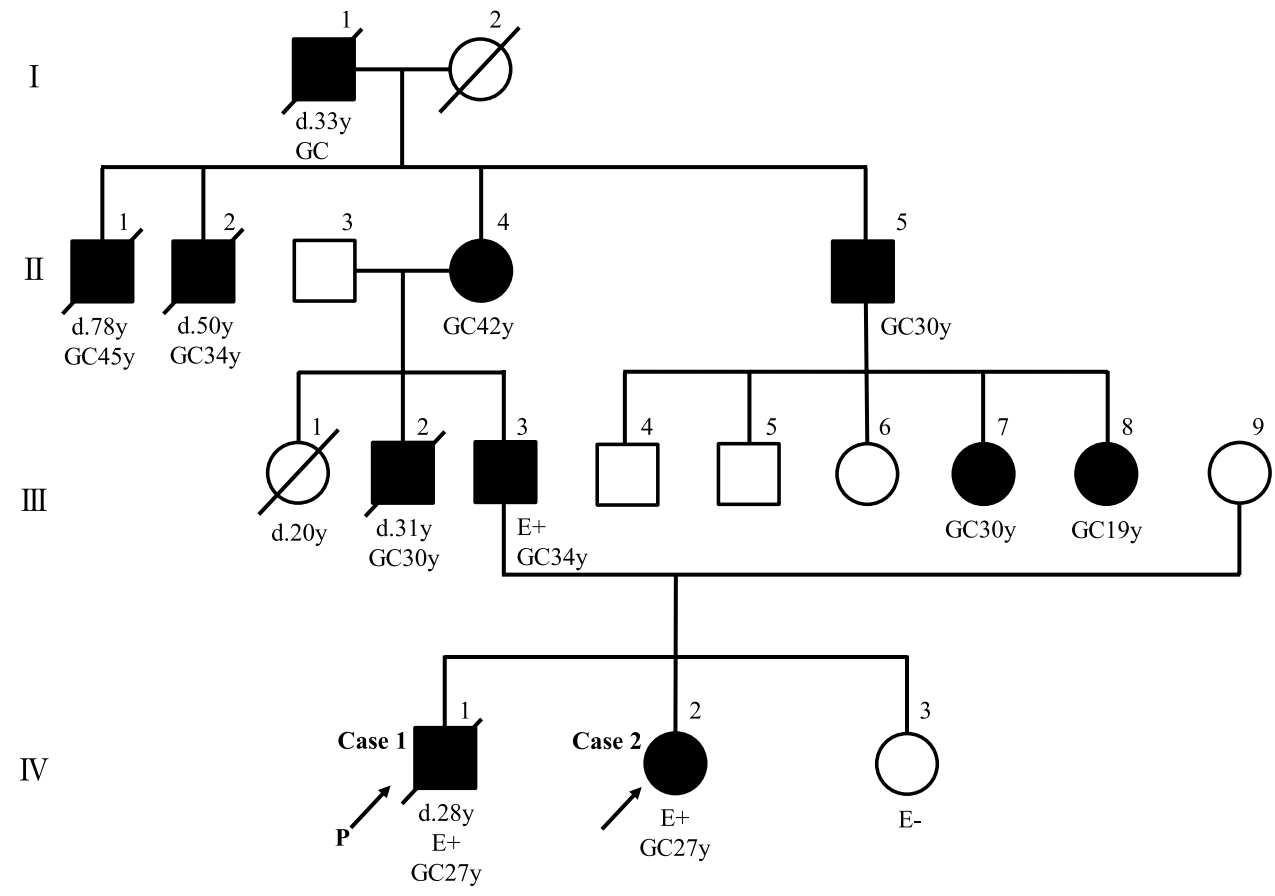

Table 1 Somatic mutations present in an intramucosal signet ring cell carcinoma (Lesion 1 of Case 2)

\begin{tabular}{|c|c|c|c|c|c|c|c|c|}
\hline Gene & $\mathrm{Chr}^{\mathrm{a}}$ & Start & Reference & Alternate & Mutation type & Amino acid change & VAF1 & VAF2 \\
\hline OR8J3 & 11 & 55904558 & $\mathrm{~T}$ & $\mathrm{C}$ & Nonsynonymous & p.T213A & 0.11 & 0.019 \\
\hline SARMI & 17 & 26712207 & - & TG & Frameshift insertion & p.L514fs & 0.109 & 0.019 \\
\hline $\mathrm{CDH1}$ & 16 & 68842705 & $\mathrm{~T}$ & $\mathrm{C}$ & Nonsynonymous & p.L214P & 0.092 & 0.018 \\
\hline YTHDF1 & 20 & 61828083 & G & A & Nonsynonymous & p.R553W & 0.085 & 0.032 \\
\hline$T T N$ & 2 & 179414419 & $\mathrm{~T}$ & $\mathrm{C}$ & Nonsynonymous & p.Q21612R & 0.082 & 0.020 \\
\hline MYSM1 & 1 & 59147930 & G & A & Synonymous & p.F262F & 0.058 & 0.030 \\
\hline VPS36 & 13 & 52990060 & - & A & Splicing & - & 0.031 & 0.004 \\
\hline
\end{tabular}

The average depth of coverage: 164

$V A F 1$ variant allele frequency in resected specimen, $V A F 2$ variant allele frequency in biopsy sample

${ }^{\mathrm{a} C h r o m o s o m e}$

mutated oncogenes in gastric cancer. These results are in agreement with the Cancer Genome Atlas database, which shows that $\mathrm{CDH} 1$ mutations are often observed in 'genomically stable' groups characterized by a diffuse histological subtype with a low frequency of genetic mutations, including in TP53 [17].

The $H p$ infection status of the patients in this study deserves attention. Both Cases 1 and 2 had similar earlyonset HDGC, the difference being that Case 1 was $\mathrm{Hp}$ infected and had advanced-stage cancer while Case 2 was $\mathrm{Hp}$-uninfected and had intramucosal SRCCs. It has been reported that various latent mutations including some in TP53 accumulate in the gastric mucosa of patients with $H p$ infection [18]. Unfortunately, we could not perform genetic analysis of the tumor tissue of Case 1 because of the small amount of DNA available. However, we speculate that some additional driver mutations such as TP53 mutation caused by $\mathrm{Hp}$ infection contributed to the acquisition of the invasive phenotype of the cancer in Case 1. In a mouse model, E-cadherin deficiency alone is sufficient to develop the gastric SRCC $[19,20]$. A recent study clarified that stem cell-specific E-cadherin deficiency alone could develop SRCC foci transiently and additive Helicobacter felis infection could maintain these foci [21]. However, it is unknown whether these foci could acquire an invasive and metastatic phenotype in the future. On the other hand, it has been reported that invasive and metastatic diffuse-type gastric cancer developed in stem cell or parietal cell-specific $C d h 1$ and Trp53 double-knockout mice [21, 22]. Our report involving two siblings with HDGC may strongly support the results of previous animal experiments. 
In Japan, gastric cancer is the most common cancer and the third leading cause of cancer death. Although most gastric cancers in Japan are caused by $H p$ infection [23], the prevalence of $H p$ infection in Japan has declined in recent years $[24,25]$ and the relative frequency of $H p$-uninfected gastric cancer may be increasing. Recently, cases of mucosal SRCC without $H p$ infection are accumulating [26, 27]. Interestingly, the histopathology of Case 2 closely resembled a typical case of $H p$-uninfected SRCC. In this regard, the results of the genetic analysis in Case 2 may be informative for clarifying the mechanism of carcinogenesis of $\mathrm{Hp}$ uninfected SRCC. However, further studies are required to identify the genetic alterations in sporadic $H p$-uninfected gastric cancer.

In conclusion, we report a family with HDGC containing two members with early-onset gastric cancer diagnosed at different stages who had different $H p$ infection status. Our results of the genetic analysis demonstrated that $\mathrm{CDH}$ mutation was the only active driver mutation in $\mathrm{Hp}$-uninfected intramucosal SRCC.

Acknowledgements This research was supported by AMED under Grant Number JP18ck0106268 by National Cancer Center Research and Development Fund 28-A-1.

\section{Compliance with ethical standards}

Conflict of interest The authors declare that they have no conflict of interest.

Ethical standards This study was approved by the ethics committee of Kyoto University.

\section{References}

1. Oliveira C, Pinheiro H, Figueiredo J, Seruca R, Carneiro F. Familial gastric cancer: genetic susceptibility, pathology, and implications for management. Lancet Oncol. 2015;16:e60-70.

2. Hansford S, Kaurah P, Li-Chang H, Woo M, Senz J, Pinheiro H, et al. Hereditary diffuse gastric cancer syndrome: $\mathrm{CDH} 1$ mutations and beyond. JAMA Oncol. 2015;1:23-32.

3. Huntsman DG, Carneiro F, Lewis FR, MacLeod PM, Hayashi A, Monaghan KG, et al. Early gastric cancer in young, asymptomatic carriers of germ-line E-cadherin mutations. N Engl J Med. 2001;25:1904-9.

4. Charlton A, Blair V, Shaw D, Parry S, Guilford P, Martin IG. Hereditary diffuse gastric cancer: predominance of multiple foci of signet ring cell carcinoma in distal stomach and transitional zone. Gut. 2004;53:814-20.

5. Sugimoto S, Yamada H, Takahashi M, Morohoshi Y, Yamaguchi $\mathrm{N}$, Tsunoda $\mathrm{Y}$, et al. Early-onset diffuse gastric cancer associated with a de novo large genomic deletion of $\mathrm{CDH} 1$ gene. Gastric Cancer. 2014;17:745-9.

6. Yamada M, Fukagawa T, Nakajima T, Asada K, Sekine S, Yamashita $S$, et al. Hereditary diffuse gastric cancer in a Japanese family with a large deletion involving $\mathrm{CDH} 1$. Gastric Cancer. 2014;17:750-6.
7. Yamada H, Shinmura K, Ito H, Kasami M, Sasaki N, Shima $\mathrm{H}$, et al. Germline alterations in the $\mathrm{CDH} 1$ gene in familial gastric cancer in the Japanese population. Cancer Sci. 2011;102:1782-8.

8. van der Post RS, Vogelaar IP, Carneiro F, Guilford P, Huntsman D, Hoogerbrugge $\mathrm{N}$, et al. Hereditary diffuse gastric cancer: updated clinical guidelines with an emphasis on germline $\mathrm{CDH} 1$ mutation carriers. J Med Genet. 2015;52:361-74.

9. Yoshida K, Sanada M, Shiraishi Y, Nowak D, Nagata Y, Yamamoto R, et al. Frequent pathway mutations of splicing machinery in myelodysplasia. Nature. 2011;478:64-9.

10. Molinaro V, Pensotti V, Marabelli M, Feroce I, Barile M, Pozzi S, et al. Complementary molecular approaches reveal heterogeneous $\mathrm{CDH} 1$ germline defects in Italian patients with hereditary diffuse gastric cancer (HDGC) syndrome. Genes Chromosomes Cancer. 2014;53:432-45.

11. Suriano G, Yew S, Ferreira P, Senz J, Kaurah P, Ford JM, et al. Characterization of a recurrent germ line mutation of the E-Cadherin gene: implications for genetic testing and clinical management. Clin Cancer Res. 2005;11:5401-9.

12. Kuboki Y, Yamashita S, Niwa T, Ushijima T, Nagatsuma A, Kuwata $\mathrm{T}$, et al. Comprehensive analyses using next-generation sequencing and immunohistochemistry enable precise treatment in advanced gastric cancer. Ann Oncol. 2016;27:127-33.

13. Mateus AR, Simões-Correia J, Figueiredo J, Heindl S, Alves CC, Suriano G, et al. E-cadherin mutations and cell motility: a genotype-phenotype correlation. Exp Cell Res. 2009;315:1393-402.

14. Wang K, Yuen ST, Xu J, Lee SP, Yan HH, Shi ST, et al. Wholegenome sequencing and comprehensive molecular profiling identify new driver mutations in gastric cancer. Nat Genet. 2014:46:573-82.

15. Kakiuchi M, Nishizawa T, Ueda H, Gotoh K, Tanaka A, Hayashi A, et al. Recurrent gain-of-function mutations of RHOA in diffuse-type gastric carcinoma. Nat Genet. 2014;46:583-7.

16. Wong SS, Kim KM, Ting JC, Yu K, Fu J, Liu S, et al. Genomic landscape and genetic heterogeneity in gastric adenocarcinoma revealed by whole-genome sequencing. Nat Commun. 2014;5:5477.

17. Cancer Genome Atlas Research Network. Comprehensive molecular characterization of gastric adenocarcinoma. Nature. 2014;513:202-9.

18. Shimizu T, Marusawa H, Matsumoto Y, Inuzuka T, Ikeda A, Fujii Y, et al. Accumulation of somatic mutations in TP53 in gastric epithelium with Helicobacter pylori infection. Gastroenterology. 2014;147:407-17.e3.

19. Humar B, Blair V, Charlton A, More H, Martin I, Guilford P. E-cadherin deficiency initiates gastric signet-ring cell carcinoma in mice and man. Cancer Res. 2009;69:2050-6.

20. Mimata A, Fukamachi H, Eishi Y, Yuasa Y. Loss of E-cadherin in mouse gastric epithelial cells induces signet ring-like cells, a possible precursor lesion of diffuse gastric cancer. Cancer Sci. 2011;102:942-50.

21. Hayakawa Y, Ariyama H, Stancikova J, Sakitani K, Asfaha S, Renz BW, et al. Mist1 expressing gastric stem cells maintain the normal and neoplastic gastric epithelium and are supported by a perivascular stem cell niche. Cancer Cell. 2015;28:800-14.

22. Shimada S, Mimata A, Sekine M, Mogushi K, Akiyama Y, Fukamachi H, et al. Synergistic tumour suppressor activity of E-cadherin and p53 in a conditional mouse model for metastatic diffuse-type gastric cancer. Gut. 2012;61:344-53.

23. Matsuo T, Ito M, Takata S, Tanaka S, Yoshihara M, Chayama K. Low prevalence of Helicobacter pylori-negative gastric cancer among Japanese. Helicobacter. 2011;16:415-9.

24. Kamada T, Haruma K, Ito M, Inoue K, Manabe N, Matsumoto H, et al. Time trends in Helicobacter pylori infection and atrophic gastritis over 40 years in Japan. Helicobacter. 2015;20:192-8. 
25. Okuda M, Osaki T, Lin Y, Yonezawa H, Maekawa K, Kamiya S, et al. Low prevalence and incidence of Helicobacter pylori infection in children: a population-based study in Japan. Helicobacter. 2015;20:133-8.

26. Yamamoto Y, Fujisaki J, Omae M, Hirasawa T, Igarashi M. Helicobacter pylori-negative gastric cancer: characteristics and endoscopic findings. Dig Endosc. 2015;27:551-61.
27. Horiuchi Y, Fujisaki J, Ishizuka N, Omae M, Ishiyama A, Yoshio $\mathrm{T}$, et al. Study on clinical factors involved in Helicobacter pyloriuninfected, undifferentiated-type early gastric cancer. Digestion. 2017;96:213-9. 